\title{
In reply: Is propofol more neurotoxic in the developing brain?
}

\author{
Tsuyoshi Tagawa $\cdot$ Shigeki Sakuraba $\cdot$ Akira Mizoguchi
}

Received: 19 September 2014 / Accepted: 19 September 2014 / Published online: 10 January 2015

(C) Japanese Society of Anesthesiologists 2014

Keywords Propofol $\cdot$ Neuroapoptosis $\cdot$ Pediatric anesthesia

To the Editor:

We thank Drs. Yu and Sun for their interest and comments regarding our recent manuscript [1]. First, we do not know whether the combination of intravenous anesthetics and sevoflurane induces disturbances in oxygenation and circulation that cannot be monitored by $\mathrm{SpO}_{2}$ and $\mathrm{CBF}$ monitoring systems. However, we believe that the degree of hypoxia and hemodynamic instability that such monitoring systems cannot detect is unlikely to influence the survival of neural cells.

Second, while MAC determination for volatile anesthetics is possible in immature mice, the same would be much more difficult for intravenous anesthetics, because a constant plasma and brain concentration would have to be achieved in order to do so. In our study, $10 \mathrm{mg} / \mathrm{kg}$ of propofol and $5 \mathrm{mg} / \mathrm{kg}$ of thiopental produced a similar degree of anesthetic depth (transient sluggishness). Therefore, the doses of both anesthetics are speculated to be approximately equipotent in immature mice.

Conflict of interest None.

\section{Reference}

1. Tagawa T, Sakuraba S, Kimura K, Mizoguchi A. Sevoflurane in combination with propofol, not thiopental, induces a more robust neuroapoptosis than sevoflurane alone in the neonatal mouse brain. J Anesth. 2014. doi:10.1007//s00540-014-1822-x.

T. Tagawa $(\bowtie) \cdot$ A. Mizoguchi

Department of Neural Regeneration and Cell Communication,

Mie University Graduate School of Medicine, 2-174 Edobashi,

Tsu, Mie 514-8507, Japan

e-mail: tsuyoshitagawa@gmail.com

S. Sakuraba

Department of Anesthesiology, Clinical Care Medicine,

Kanagawa Dental College, Yokosuka, Kanagawa, Japan 